\title{
EXPERIMENTACIÓN COMERCIAL PARA LA MEJORA DEL SEO LOCAL DE UNA CADENA DE DISTRIBUCIÓN ALIMENTARIA.
}

\author{
Rocio Lopez-Navarro, Maria Muñoz-Martinez, Carmen Escriba-Perez, Juan Manuel Buitrago- \\ Vera*
}

Universitat Politècnica de València (València, jmbuitrago@esp.upv.es)

\section{Resumen}

Hoy en día es muy importante que las empresas trabajen sus sitios web en busca de mejorar su posición en los buscadores (Google, Yahoo, etc.), para ello emplean una serie de técnicas y herramientas que aumentan la visibilidad del establecimiento o negocio según su situación geográfica (SEO local). El objetivo del trabajo es realizar un experimento para observar la evolución del SEO local de las ubicaciones de una cadena de supermercados en la ciudad de València en los servicios de búsqueda y mapas de Google. Para ello se seleccionaron 53 supermercados de los que Google no disponía fotografías propias y se creó un grupo control al que no se les aplicó ningún tratamiento. Este grupo estaba formado por 14 supermercados, seleccionados en base a criterios geográficos (distritos de la ciudad de València), características propias de los locales (metros cuadrados, parking propio) y densidad comercial. Como indicadores de evolución se usaron las visualizaciones (de la búsqueda o de Maps), las búsquedas (directas e indirectas) y acciones (llamada, visita al sitio web y solicitud de indicaciones). Se observó una mejora en los tres indicadores con una repercusión de 3.5 millones de visualizaciones, 3,3 millones de búsquedas y 1,9 millones de acciones de los usuarios más que en 2019.

Palabras Clave: SEO Local, Posicionamiento, Supermercados, Distribución alimentaria.

\section{Introducción y objetivos}

El marketing digital es la aplicación de las estrategias de comercialización llevadas a cabo en los medios digitales. En el ámbito digital aparecen nuevas particularidades como la inmediatez (Hernandez et al, 2017).

Hoy en día, en un mundo en el que las personas estamos constantemente conectadas a la web es importante que las empresas centren gran parte de sus esfuerzos en desarrollar su imagen dentro de Internet. Por ello, las empresas deben utilizar herramientas y técnicas que les permitan posicionarse en los mejores puestos de los buscadores. Estos posicionamientos en los motores de búsqueda se conocen como Search Engine Optimization (SEO) (Prat, 2016).

El objetivo de este trabajo es el estudio de una aplicación real de estas técnicas y herramientas, en este caso en concreto se introdujeron fotografías que mejorarán la ficha de Google Maps de cada uno de los supermercados elegidos de la ciudad de València de una conocida cadena de distribución alimentaria de la Comunitat Valenciana. Por ello, se puede observar cómo una empresa puede mejorar su SEO, concretamente el SEO local, es decir, el que se refiere a su zona geográfica.

El desarrollo experimental de este trabajo fue llevado a cabo durante gran parte del confinamiento a causa de la pandemia de COVID-19, lo que según el estudio de movilidad local sobre COVID-19 realizado por Google (Google, 2020) muestra que los usuarios se han desplazado a supermercados de su ámbito local, algo que justifica la realización de este estudio dada la importancia que ha tenido el posicionamiento local durante este periodo.

\section{Metodología}

Para llevar a término este estudio se diseñó un experimento comercial que incluía a los 53 supermercados de la ciudad de València que no contenían fotografías propias en su ficha de ubicación. Estos establecimientos se separaron en dos grupos: uno al que se le incluyeron fotografías a las fichas de ubicación (Grupo Tratamiento- GT) y otro al que no se le aplicó ningún cambio (Grupo Control- GC). La selección del GC se realizó en base a tres criterios: geográfico, en función del distrito de la ciudad de València donde se encontraban; número de metros cuadrados del local; y la densidad comercial, que en este caso se definió como el número de supermercados de la competencia que se encontraban en un radio de $500 \mathrm{~m}$. Una vez definidos los grupos de estudio se comenzó con la toma de datos previa al tratamiento que fueron: el número de visualizaciones, búsquedas (directas e indirectas) y acciones de los usuarios en cada ubicación, semanalmente durante 3 meses (12 semanas). Una vez pasado ese periodo de tiempo se añadieron las fotografías a las ubicaciones del GT y se tomaron datos semanalmente durante 21 semanas más para su comparación con el periodo de 3 meses previo y así poder evaluar los resultados que fueron calculados mediante el incremento del GT con respecto al GC en los periodos previo y posterior a la adición de fotografías. 


\section{Resultados}

Respecto al impacto en las visualizaciones (Gráfico 1) se observa cómo en las semanas anteriores a la introducción de las fotografías, el incremento estaba cayendo hasta llegar a puntos negativos, de manera que, en promedio, los establecimientos de Control empezaban a superar en visualizaciones a los de Tratamiento. A partir de la semana 14, tras la introducción de las fotografías y durante el periodo de latencia, la situación se empieza a estabilizar. Sin embargo, no es hasta la semana 24 cuando se empiezan a notar los resultados y los supermercados de Tratamiento empiezan a superar a los de "Control" en promedio de visualizaciones, llegando a alcanzar en la semana 31 un incremento del 12,23\% respecto al grupo de Control, por lo que en promedio se observó una mejora de un 5,49\%

Gráfico 1. Visualizaciones, diferencia Tratamiento-Control

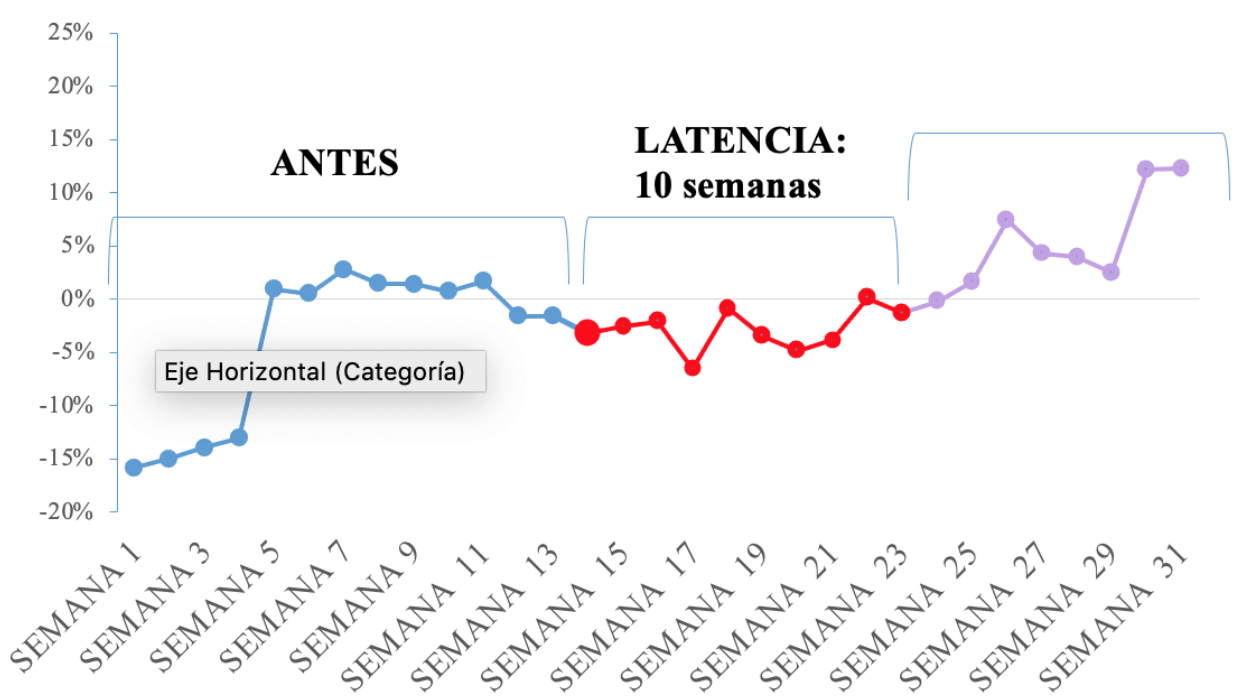

Fuente: Elaboración propiá a partir de los datos de la aplicación Google My Business

En cuanto a las búsquedas, tanto directas como indirectas, antes de agregar las fotografías se aprecia una situación de estabilidad a partir de la semana 5 (Gráfico 2), sin embargo, el incremento es negativo lo que implica que en promedio los supermercados de GC aumenten en mayor medida que los de GT. Tras la introducción de las fotografías en Google Maps, encontramos un periodo de latencia, pero a partir de la semana 22, la diferencia Tratamiento-Control se va reduciendo, hasta que a partir de la semana 24 se vuelve positiva, suponiendo una mejora de un $7 \%$.

Gráfico 2. Búsquedas, diferencia control-tratamiento

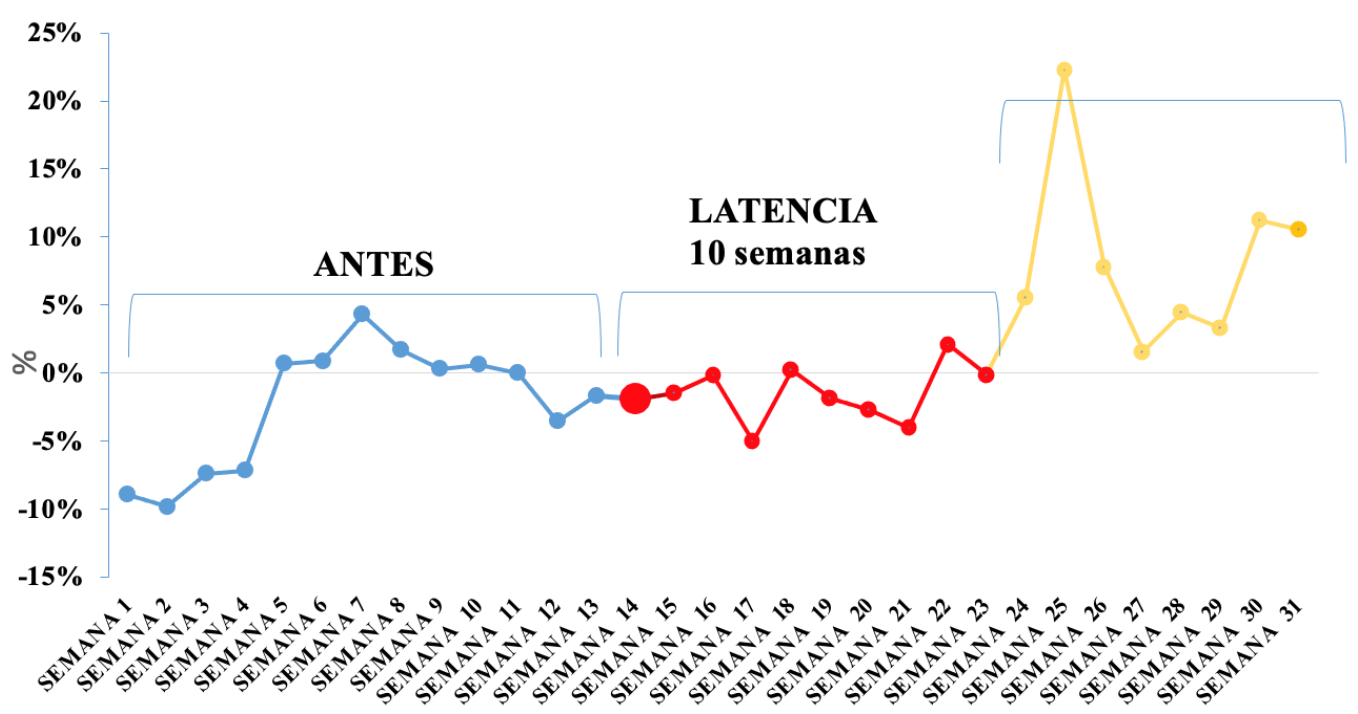

Fuente: Elaboración propia a partir de los datos de la aplicación Google My Business. 
Por último, tal y como muestra el Gráfico 3 en las acciones se observa un comportamiento muy similar al de las visualizaciones y las búsquedas con un periodo de latencia de 10 semanas hasta observar una mejora a partir de la semana 24 del experimento, resultando en una mejora positiva de un $8,3 \%$.

Gráfico 3. Acciones de los usuários diferencia Control-Tratamiento

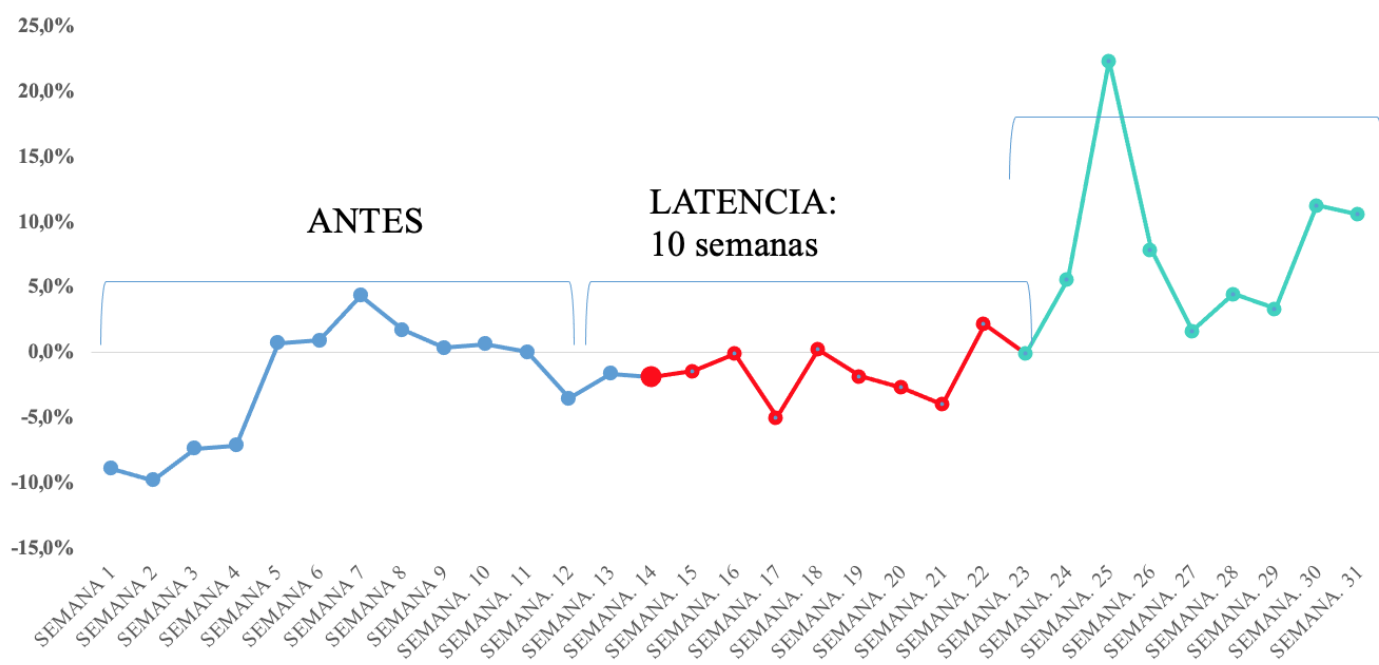

Fuente: Elaboración propia a partir de los datos de la aplicación Google My Business.

\section{Conclusiones}

El experimento comercial llevado a cabo en los supermercados que tiene presente una cadena de supermercados en la ciudad de València ha resultado en una mejora de la visibilidad local en internet mediante la adición de fotografías de los locales. Concretamente, extrapolando los resultados obtenidos a un año completo, con estas mejoras se esperarían 3,5 millones de visualizaciones más que si no se hubieran añadido fotografías, 3,3 millones de búsquedas más y 1,9 millones de acciones de los usuarios, sólo en la ciudad de València.

Cabe destacar que dado el contexto de confinamiento de COVID-19 en el que se realizó gran parte del experimento comercial y como bien evidencia el estudio de movilidad local sobre COVID-19 realizado por Google (Google, 2020), donde muestra una reducción de la afluencia de personas a supermercados pese a que se mantuvieron abiertos durante todo el confinamiento, todo ello ha podido influir en las cifras obtenidas, pudiendo ser ligeramente superior en condiciones sin pandemia.

Pese a ello, se puede concluir que es recomendable realizar este tipo de acciones y estudios sobre SEO Local en la distribución alimentaria que, además de no suponer un gran coste para la empresa, supone una mejorar en su posicionamiento en web y a nivel local en su área de influencia, que puede tener un impacto positivo en las ventas.

\section{Bibliografía}

Google. (2020). Informes de Movilidad Local sobre el COVID-19. Recuperado de https://www.google.com/covid19/mobility/ [Consultado por última vez: 16 de julio de 2020]

Hernández, M.A., Estrade, J.M. y Jordán, D. (2017). Marketing Digital Mobile Marketing, SEO y Analítica web. Madrid: Anaya Multimedia

Prat, M. (2016). Posicionamiento web: estrategias de SEO: Google y otros buscadores. Barcelona: Ediciones ENI. 